\title{
Autopercepción del Profesorado y Equipo Directivo en el Desarrollo de la Creatividad. Un Estudio de Caso Valorado con VADECRIE
}

\author{
Autoperception of Teachers and Direct Team in the \\ Development of Creativity. A Case Study Analyzed with \\ VADECRIE
}

\author{
Saturnino de la Torre ${ }^{1 *}$ \\ José Luis Marcilla Cebrián ${ }^{2}$ \\ Ana María de la Torre Frade ${ }^{3}$ \\ ${ }^{1}$ Universidad de Barcelona, España \\ ${ }^{2}$ Skola Klic S.R.O., República Checa \\ ${ }^{3}$ INS Escuela de Hotelería y Turismo de Barcelona, España
}

\begin{abstract}
El objetivo primordial de este trabajo es constatar la autopercepción que tiene el profesorado y equipo directivo de la creatividad en escuela. Valorar hasta qué punto existe un desarrollo creativo de la institución educativa a partir de un estudio de caso. Para ello aplicamos varios parámetros del instrumento "Valoración del desarrollo creativo de instituciones educativas" (VADECRIE) referidos a la dimensión humana: profesorado, equipo directivo y estudiantes. Intentamos conocer sus opiniones y autopercepciones. De ese modo triangularemos la información proveniente de dichas fuentes. La investigación se plantea desde un enfoque o paradigma de la complejidad y se utiliza como metodología el estudio de caso, centrando en el Colegio concertado Santo Ángel de la Guarda (Albacete. España). Este estudio se realizó con 348 participantes, de los cuales 14 fueron docentes de enseñanza básica, 7 miembros del equipo directivo y 327 alumnos. Los resultados ponen de manifiesto que existe una autopercepción positiva de la creatividad en el Centro.
\end{abstract}

Descriptores: Creatividad; Desarrollo creativo; Escuelas creativas; Autopercepción creativa; Pensamiento del profesor.

\begin{abstract}
The main objective of this work is to verify the self-perception of the faculty and management team of creativity in school. Evaluate the extent to which there is a creative development of the educational institution from a case study. To do this we apply several parameters of the instrument "Valuing the creative development of educational institutions" (VADECRIE) referring to the human dimension: teaching staff, management team and students. We try to know their opinions and selfperceptions. In this way, we will triangulate and contrast the information coming from these sources. The research is based on an approach or paradigm of complexity and is used as a case study methodology, focusing on the Colegio Santo Ángel de la Guarda (Albacete, Spain). This study was carried out with 348 participants, of which 14 were teachers of basic education, 7 members of the management team and 327 students. The results show that there is a positive self-perception of creativity in the Center.
\end{abstract}

Keywords: Creativity; Creative development; Creative schools; Creative selfperception; Thought of the professor.

*Contacto: sentipensar@yahoo.es

ISSN: 2254-3139

www.rinace.net/riejs/

revistas.uam.es/riejs
Recibido: $\quad 15$ de marzo 2018

$1^{\text {a }}$ Evaluación: 14 de junio 2018

$2^{\text {a }}$ Evaluación: 3 de septiembre 2018

Aceptado: 2 de octubre 2018 


\section{Introducción}

En las últimas décadas, la sociedad actual está viviendo una serie de trasformaciones vertiginosas en el ámbito tecnológico, laboral, sociocultural, generando nuevas demandas que necesita satisfacer. Esto ha traído consigo la aparición de procesos adaptativos que no siempre resultan fáciles de asimilar, sobre todo por el temor, la ansiedad y la inseguridad que generan los cambios. No obstante, la sociedad les ha podido hacer frente gracias a sus propios impulsos creativos, los cuales les han permitido abordar este momento de evolución que está viviendo (Torre y Rodríguez, 2009). La intención de este artículo es seguir contribuyendo a esta ola de cambio, de transformación hacia instituciones educativas con planteamientos y prácticas más innovadoras y creativas.

Los sistemas educativos, por su parte, siguen influidos por modelos basados en la instrucción y transmisión del conocimiento, valorándose más los conocimientos y rendimientos que la formación integral de las personas (Torre, 2012). Sin Embargo, cada vez son más las escuelas e instituciones comprometidas con una nueva forma de aprender, una forma abierta, comprensiva, integradora y transformadora. "Es preciso preparar a los estudiantes para el cambio mediante el desarrollo de la capacidad creativa que dé soluciones concretas a los desafíos actuales" (Menchén, 1998, p. 13).

Así mismo, debemos hacer frente a la problemática de la desorientación del profesorado, que termina por buscar el camino fácil con el libro de texto, sin tomar ninguna iniciativa creativa. Pero si el componente creativo apenas ha contado durante la preparación y selección del profesorado, lo extraño sería que estas medidas creativas se reflejaran en el aula. Es por ello que necesitamos dotar de experiencias innovadoras a los centros, transformando así la educación y dando lugar a la creación de comunidades abiertas de aprendizaje. La Red Internacional de Escuelas Creativas (RIEC) proporciona, a través de publicaciones y Encuentros, experiencias valiosas de Creatividad, Ecoformación y ejemplos de Proyectos creativos ecoformadores (Pitz, Silva y Silva, 2017; Torre y Violant, 2016;). La vivencia de experiencias creativas es una estrategia eficaz para el cambio de conciencia (Costa et al., 2016).

Así pues, los cambios y las innovaciones son respuestas a una necesidad adaptativa de los Centros escolares a las exigencias sociales, institucionales y personales del propio centro. No hay crecimiento sin cambios. Los cambios y las innovaciones, finalmente, también son necesarios para satisfacer las expectativas de las personas. Por ello, pretendemos constatar la autopercepción creativa del equipo directivo, profesorado y alumnado respecto al modo cómo se desarrolla la actividad educativa del Centro, dentro y fuera del aula. Para ello nos valemos del Instrumento VADECRIE (Valoración del desarrollo creativo del centro educativo), descrito por S. Torre (2012) que nos proporciona indicadores del modo de pensar, sentir y actuar de los principales agentes educativos. La cultura innovadora de un centro, además de parámetro de desarrollo creativo, nos informa del potencial transformador del mismo.

\section{Revisión de la literatura}

Disponemos de una amplísima literatura sobre la creatividad en general y sus aplicaciones a la educción, la empresa, el marketing, pero poca referida a las instituciones educativas. Es por ello que la fundamentación de la creatividad institucional hemos de buscarla en las organizaciones que aprenden, a través de la visión de la creatividad compleja y 
transdisciplinar que vaya más allá de los procesos psicológicos y personales y en un modo de educar basado en el desarrollo humano más que en el rendimiento académico. En tal sentido, la enseñanza a través de Proyectos creativos ecoformadores (Pitz, Silva y Silva, 2017; Torre y Zwierewicz, 2009), de Diálogos analógicos creativos (Torre, 2014) de entornos y escenarios de autoaprendizaje, de solución creativa de problemas... son modalidades que encontramos en las aulas y centros creativos.

En el acta de creación de RIEC, llevada a cabo en el Fórum de Innovación y Creatividad de 2012, se fundamenta la Red "sobre los principios de sustentabilidad, ecología de saberes e integración institucional del conocimiento, superando de ese modo la endémica fragmentación del conocimiento". En la enseñanza se busca la superación de la fragmentación disciplinar de las asignaturas por propuestas más integradoras en forma de proyectos, talleres, espacios o escenarios de trabajo, estrategias creativas, teniendo como bases teóricas el "Decálogo sobre Transdisciplinariedad y Ecoformación" (Torre et al., 2011). Estos serían algunos de los principios que dan sentido y fundamento a este trabajo.

Según Torre y Violant (2016), el Movimiento de Escuelas creativas, tiene sus referentes teóricos en: a) La escuela como organización viva que aprende y se transforma; 2) La creatividad desde una perspectiva compleja y psicosocial; 3) Los valores y el desarrollo humano como referentes, frente al discurso del desarrollo académico tan extendido; y 4) El pensamiento complejo, Transdisciplinariedad y ecología de saberes, superando de ese modo la endémica fragmentación del conocimiento.

Nos remitimos en este aspecto de fundamentación a las recientes publicaciones de autores como Pujol, Moraes, Torre, Menchén, en la obra digital coordinada por Pujol, Torre y Lorenzo (2015) titulada "Escuelas y aulas creativas. Reflexiones y experiencias". Los autores mencionados abordan cuestiones relacionadas con la creatividad desde una mirada compleja y transdisciplinar, el acento puesto en el desarrollo humano frente al discurso académico, el concepto de escuelas galácticas como expresión de una mirada planetaria de la educación, abierta al futuro, cargada de valores humanos socio ambientales y trascendentes.

Pero las escuelas, en tanto que organizaciones vivas, forman parte de contextos sociopolíticos y por tanto no siempre los ideales educativos llegan a internalizarse. Los Centros educativos, como cualquier otra organización, están sometidos a las presiones y requerimientos políticos del momento. Adaptarse al entorno y responder a demandas nuevas es uno de los retos ineludibles de las instituciones que desean actuar satisfactoriamente. Las soluciones viejas suelen servir muy poco para las situaciones nuevas. Esa adaptación y ajuste continuos conllevan un ejercicio de análisis permanente de la práctica que permite orientar de forma adecuada las soluciones que requiere cada uno de los ámbitos de gestión en cada caso.

Así pues, los cambios y las innovaciones son respuestas a una necesidad adaptativa de los centros escolares a las exigencias sociopolíticas, psicodidácticas, institucionales y ambientales y del propio centro en su enclave. No hay crecimiento sin cambios. Los cambios y las innovaciones, finalmente, también son necesarios para el desarrollo personal y profesional del profesorado. 
Torre (2012) entiende las escuelas o centros educativos creativos como:

Organizaciones vivas que van más allá de donde parten (trascienden), que dan más
de lo que tienen y sobrepasan lo que de ellas se espera (recrean), que reconocen lo mejor
de sus alumnos y profesorado (valoran), que crecen por dentro y por fuera buscando
en todo la calidad y la mejora (transforman). En otras palabras, aquellos centros que
desarrollan los potenciales creativos de los escolares, valores humanos, sociales, de
convivencia, libertady creatividad. (p. 59)

Dicho autor enumera diez parámetros para describir a las instituciones educativas creativas, que luego agrupa en tres ámbitos o dimensiones: los referidos al potencial humano (profesorado y alumnado, equipo directivo, espíritu emprendedor); los referidos al nivel de conciencia creativa (cultura innovadora, creatividad como valor, visión transdisciplinar, valores humanos); los referidos al plan estratégico y de acción (currículo polivalente, metodología diversificada, evaluación formadora y emergente).

En este trabajo nos centraremos en el potencial humano, que caracterizamos con los siguientes rasgos:

- Liderazgo estimulador y creativo, entendido como la capacidad de la persona que está al frente de la institución para favorecer tanto el desarrollo y crecimiento institucional como de las personas que integran la misma, con actitud positiva y de reconocimiento. La dirección creativa adopta una actitud dialogante y comprometida, impulsando y facilitando proyectos innovadores y creativos.

- Profesorado creativo, entendido como aquel que tiene una alta autopercepción de la creatividad y es capaz de trasmitir actitudes creativas, de entusiasmar, inspirar y dejar huella constructiva en el alumnado. Les distingue su actitud abierta, flexible, colaborativa, dialogante y emprendedora. Conciben la educación en términos de competencias para la vida. Aprende de los errores y utiliza estrategias diversas. En dos palabras, posee un espíritu creativo.

- Espíritu emprendedor y de iniciativa. Los miembros de la comunidad educativa conciben la educación y la creatividad como instrumentos de transformación personal, institucional y social, promoviéndose valores como; autoconfianza e independencia, persistencia, sentido de la responsabilidad, fijar metas, etc.

Autores como Gómez y Machado (2010) en su obra "Organizaciones educativas creativas", Menchén (2015) en "La necesidad de escuelas creativas", Robinson y Aronica (2015) en su obra "Escuelas creativas", el Proyecto de Escuelas Creativas de Telefónica utilizando la metodología eBulli del cocinero Ferrán Adrià, son indicadores clave de la necesidad incorporar la creatividad en la coyuntura socioeducativa actual. Al tiempo que ponen de manifiesto cómo las escuelas tradicionales matan la creatividad, muestran ejemplos de escuelas e institutos que promueven la creatividad. Encontramos experiencias en muchos países. También encontramos Escuelas creativas reconocidas como tales por RIEC en Goiânia, Tocantins, Sao Paulo, Blumenau (en Brasil), Barcelona o Lima. Todas ellas pertenecen a RIEC.

Digamos, para concluir, que estamos ante un nuevo enfoque de la enseñanza y de la innovación, entendida como un proceso dinámico y abierto, de carácter multidimensional y complejo, inserto en una realidad sociocultural y humana que busca el crecimiento personal, institucional y mejora social, por lo que requiere estrategias de participación colaborativa (Torre, 2012). Esta perspectiva origina, sin duda, múltiples cambios en los 
roles de quienes participan en la planificación, ejecución y evaluación de la educación; en la distribución (más equilibrada y justa) del poder; en el diseño de modelos organizativos propios; en los niveles de participación, cooperación y cohesión de los actores; en el logro de aprendizajes que posibiliten la solución de problemas pertinentes, así como la realización de variados recursos existente en el medio.

\section{Problema de investigación}

Nadie pone en duda el hecho que la escuela juega un papel fundamental en el desarrollo creativo de los alumnos. Bien podríamos convertir en dicho "Escuela creativa, alumnos creativos". Tanto Lipton (2014) como Dispenza (2014) mantienen que las estructuras mentales, emocionales y de personalidad se van conformando en los primeros años de vida. De ahí la importancia de crear ambientes estimulantes para el desarrollo de los valores humanos si queremos una sociedad más solidaria, ética y respetuosa con el medio natural y con los demás. De lo contrario, si seguimos manteniendo una escuela basada en el discurso académico, en la estandarización de resultados y ponemos el acento en el dominio de contenidos en lugar de ponerlo en las personas, sus valores y creencias, seguiremos alimentando una sociedad consumista, materialista, insolidaria con los más débiles y marginados. ¿Qué deberíamos hacer?

Los modelos tradicionales de enseñanza han podido funcionar en el pasado; pero ya no se adecúan a un mundo cambiante, global y diverso como el actual. A juicio de numerosos pensadores, sociólogos y pedagogos de nuestros días (Robinson, Laszlo, Gerver, Moraes, Thao, Morin, Menchén, por citar sólo algunos) los sistemas educativos actuales están anclados en prácticas pedagógicas propias de los siglos XIX y XX. Seguimos utilizando una metodología centrada en los resultados, priorizando los contenidos instructivos sobre las competencias transversales, actitudes y valores propios de la sociedad del conocimiento.

Tomando en cuenta lo expuesto, proponemos constatar la autopercepción del profesorado y equipo directivo como concepto que esté presente en la escuela; esto es, valorar la importancia que otorgan a la creatividad en su proceder docente o directivo. Esta autopercepción nos indicará el grado de consideración social otorgado a la creatividad que será contrastada con la percepción que tiene el alumnado de esas mismas conductas. De ese modo conseguimos triangular la información en lo que sería la vertiente humana de un Centro educativo. De ese modo podremos valorar el desarrollo humano creativo del centro objeto de estudio.

Lejos de intentar evaluar, examinar o enjuiciar el nivel de creatividad, el instrumento utilizado está diseñado para valorar la autopercepción y reconocer potencialidades, visiones y prácticas innovadoras de los agentes educativos.

Planteamos los objetivos a través de las siguientes preguntas:

- ¿Cuál es la autopercepción que tiene el profesorado de los indicadores de la creatividad expresados en el instrumento VADECRIE?

- ¿Existe concordancia entre la autopercepción docente y la que tiene el alumnado de sus actuaciones o por el contrario hay discrepancias?

- ¿Cuál es la autopercepción del equipo directivo respecto a la creatividad en el centro? 


\section{Método}

La investigación se plantea bajo el enfoque del pensamiento complejo o paradigma ecosistémicos, tal como lo describe Moraes (2004) sustentado en tres pilares: i) complementariedad de los elementos a considerar, ii) interacción entre ellos y, iii) apertura a la emergencia. La realidad educativa es como un sistema dinámico y abierto que se va construyendo en base a nuevas informaciones e interacciones dando origen a nuevas emergencias. Por tanto, se adopta una metodología de tipo cualitativo, en la que predomina la interpretación sobre el dato en sí y la intersubjetividad sobre la objetividad. Cuestiones como la autopercepción y pensamiento del profesor son realidades lábiles que han de ser abordadas desde un enfoque complejo.

En el diseño se ha utilizado el Estudio de caso (De Miguel, 2006) por responder mejor a nuestro propósito y considerarlo una herramienta de investigación valiosa cuya fortaleza radica en que a través de ella obtenemos evidencias y explicaciones relativas a la problemática planteada, a diferencia de los métodos cuantitativos sólo se centran en información verbal obtenida a través de informaciones cuantificables. De acuerdo con De Miguel (2006) el estudio de caso es un método de análisis intensivo y completo de un hecho, problema o suceso real con la finalidad de conocerlo, interpretarlo, resolverlo, generar hipótesis, contrastar datos, reflexionar, completar conocimientos, diagnosticarlo y, en ocasiones, entrenarse en los posibles procedimientos alternativos de solución.

Eisenhardt (1989, p. 542) concibe el estudio de caso contemporáneo como "una estrategia de investigación dirigida a comprender las dinámicas presentes en contextos singulares”. De hecho, el estudio de caso nos permite abordar planteamientos complejos de forma interactiva, abierta y dinámica, así como conocer en mayor profundidad un fenómeno o en nuestro caso el centro educativo por lo que respecta a la creatividad como valor institucionalizado. Nos permite conocer a los agentes educativos en sus opiniones, creencias, actitudes, valores y experiencias, que son los que conforman las matrices pedagógicas de las que nos habla Furlanetto (2011) y Torre Frade (2017).

El estudio de caso es, pues, coherente y pertinente con los objetivos del estudio al proporcionarnos una información diversifica, múltiple, contextualizada, adentrándonos en concepciones e impulsos subyacentes de docentes, alumnos y miembros del equipo directivo. Cada uno con sus intereses, roles y preferencias.

El procedimiento metodológico se desarrolló en varias etapas:

Revisión, selección y adaptación de dimensiones, descriptores y criterios: Una vez seleccionado el Centro educativo como base para la elaboración de los cuestionarios, se procedió a escoger las dimensiones, criterios y descriptores (ítems) más idóneos para la investigación. Se pretendía valorar de qué manera el equipo directivo y profesores, desde sus respectivos cargos y funciones, y los alumnos, a partir de sus creaciones, contribuyen al fomento de la creatividad en la comunidad educativa.

Elaboración y adaptación de los cuestionarios: En esta etapa se procedió a la adaptación y elaboración de los cuestionarios (anexos 1, 2 y 3). La elaboración de los mismos estuvo determinada por el instrumento de valoración del desarrollo creativo de instituciones educativas (VADECRIE) de Torre (2012), formado por 10 dimensiones y 40 ítems. El cuestionario entregado al profesorado fue elaborado por Suanno en su tesis doctoral a partir del instrumento VADCRIE de Torre (2012) por lo que atribuimos la autoría a Torre- 
Suanno. Al alumnado se le entregó una réplica del cuestionario del profesor, con el fin de obtener un punto de mira complementario.

El Cuestionario de Autopercepción creativa del profesorado y alumnado está construido en base a 10 rasgos que Torre (2012, p. 115) considera fundamentales para describir al profesor creativo. Estos son: 1) convicción, creyendo en lo que hace; 2) reconocimiento de valores y logros; 3) entusiasmo; 4) amplitud de conciencia; 5) capacidad de transcender y transformar; 6) capacidad de impactar, sorprender, incitar; 7) vitalismo, conectar con la vida; 8) originalidad, imaginación, inventiva; 9) sentipensamiento, conectando pensamiento, emoción, acción y transcendencia; y 10) trasmite valores humanos, sociales, medioambientales, planetarios. Dichos rasgos están distribuidos en el cuestionario delo 1 al 10 y reforzados con diferente lenguaje y situaciones en cuatro ítems, consiguiendo de ese modo evitar o identificar las incoherencias y lograr una mayor validez interna del cuestionario. De ese modo, los ítems 1,11, 21, 31, hacen referencia a las convicciones. Los ítems 2, 12, 22, 32 hacen referencia al reconocimiento... Los ítems 10, 20, 30, 40, confluyen en la trasmisión de valores.

Para estimar la confiabilidad del instrumento, se empleó el método de Alfa de Cronbach de consistencia interna. Este método de cálculo requiere una sola administración del instrumento de medición y permite determinar la consistencia interna del instrumento, indicando la coherencia y consistencia del desempeño del sujeto a través de las respuestas. $\mathrm{Si}$ los sujetos se desempeñan consistentemente, se dice que el instrumento tiene consistencia interna (Aiken, 1996). La medida de la fiabilidad mediante el coeficiente Alfa de Cronbach asume que los ítems miden o valoran un mismo constructo y que están altamente correlacionados (Welch y Comer, 1988). Cuanto más cerca se encuentre el valor alfa de 1, mayor es la consistencia interna de los ítems analizados. Estos ítems fueron organizados en una escala de tipo Likert con 4 categorías: Es habitual $(\mathrm{A}=4)$, Frecuentemente $(\mathrm{B}=3)$, Ocasionalmente $(\mathrm{C}=2)$, Nunca o casi nunca $(\mathrm{D}=1)$. Al eliminarse el punto intermedio obliga a situarse a un lado y otro de la escala.

Aplicando el índice Alfa de Cronbach, los niveles de confiabilidad oscilaron entre 0,851 en el cuestionario del alumnado, 0,922 en el cuestionario del profesorado y 0,906 en el equipo directivo. Estos resultados avalan la consistencia interna del instrumento.

\section{La muestra}

De acuerdo a los objetivos del estudio, la muestra o unidad de análisis está formada por los miembros del equipo directivo (directores, inspectores y orientadores), profesores y alumnos del Centro Educativo Santo Ángel de la Guarda, ubicado en el barrio de Fátima (Albacete. España). El nivel económico y social del enclave puede calificase como bueno, contando el barrio con servicios como centros de Educación Infantil y Primaria, biblioteca y ludoteca públicas, zonas deportivas municipales, entre otros. El centro se encuentra actualmente concertado y dispone de un Concierto Educativo para 2 líneas en Educación Infantil, Educación Primaria y Educación Secundaria Obligatoria.

El proceso para conseguir que respondieran a los cuestionarios no resultó fácil al considerarse voluntario. Hubo que insistir en reiteradas ocasiones y el proceso se extendió más de un mes. A pesar de las dificultades se logró reunir un total 348 respuestas.

Respecto a la representatividad del profesorado cabe resaltar: 1) respecto al sexo destaca un ligero predomino de mujeres $(57,14 \%)$. 2) El rango de edad de los docentes que tuvo mayor representación fue el de 34-43 años con 42,86\%. 3) Respecto a los años de servicio, 
la muestra presentó porcentajes similares para el primer tramo (1-11 años de docencia) y tercero (22-31 años). El porcentaje más bajo, vino representado por los docentes que llevan más de 31 años de servicio.

En el cuadro 1 se presenta la distribución del alumnado por sexo y ciclos. La muestra estuvo marcada por un ligero predominio de niñas. Respecto al Ciclo educativo al que pertenecen, la muestra presentó porcentajes similares, con una ligera mayoría en segundo ciclo, sin que pudiera hablarse de diferencia significativa.

Cuadro 1. Distribución del alumnado por sexo y ciclos

\begin{tabular}{lcc}
\hline & N $=\mathbf{3 2 7}$ & PORCENTAJE \\
\hline Niños & 154 & 47,09 \\
Niñas & 173 & 52,9 \\
\hline $1^{\circ}$ Ciclo Educación Primaria (6-7 años) & 106 & 32,42 \\
$2^{\circ}$ Ciclo Educación Primaria (8-9 años) & 114 & 34,86 \\
$3^{\circ}$ Ciclo Educación Primaria (10-1 1 años) & 107 & 32,72 \\
\hline
\end{tabular}

Fuente: Elaboración propia.

\section{Resultados}

Los datos obtenidos a través de los cuestionarios fueron analizados con el programa estadístico SPSS 15.0. Para establecer la confiabilidad de los instrumentos a través de su consistencia interna, se empleó el coeficiente Alfa de Cronbach.

\subsection{Valoración general del desarrollo creativo del Centro}

La puntuación media de toda la muestra en el instrumento VADECRIE fue de 3,4 (DT = 1,94). Con el fin de encontrar diferencias significativas en la autopercepción de la creatividad del centro por parte de los diferentes agentes educativos, se realizaron los correspondientes análisis de diferencias a través del análisis estadístico de varianza (ANOVA) para comparar varios grupos (equipo directivo, profesores y alumnado) y $t$ student para contrastar qué grupos tienen medias significativamente distintas. Al aplicar la función ANOVA, se obtiene el estadístico F con su nivel de significación $p$. Si el nivel de significación entre los grupos es menor o igual que 0,05, rechazamos la hipótesis de igualdad de medias, e interpretamos que las diferencias entre ambos grupos son significativas.

De este modo, se encontraron diferencias significativas $(\mathrm{F}=13,553, p=0,002)$ entre las puntuaciones totales obtenidas por los docentes $(3,78)$ y por el alumnado $(2,86)$. Tal y como se muestra en la figura 1 , ambos obtienen puntuaciones similares en cuanto a la categoría conciencia creativa de la institución y la categoría de Potencial Humano, sin embargo, la gran diferencia se hace patente en la categoría Plan de acción en la que la puntuación de los docentes dobla a la obtenida por los alumnos/as.

Las puntuaciones del Equipo directivo muestran que el desarrollo creativo del centro viene determinado principalmente por las metodologías utilizadas (Plan de acción) seguido muy de cerca por la Conciencia Creativa del centro. A diferencia de los docentes y los alumnos, el equipo directivo consideró que el Potencial Humano es el ámbito que menos aporta al desarrollo creativo del centro con una puntuación de 1,04. Mmientras que los docentes y alumnos consideraron que el aporte del personal a la creatividad del centro correspondía a 1,88 y 1,66 respectivamente. 


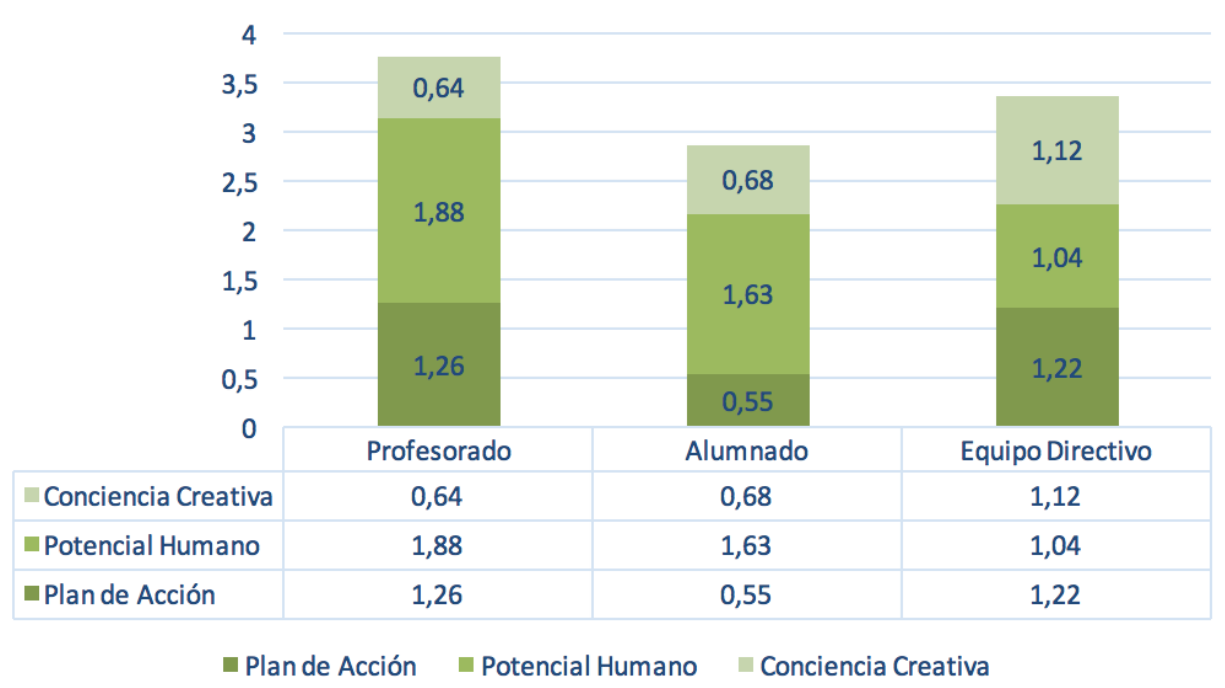

Figura 1. Valoración general del desarrollo educativo del centro

Fuente: Elaboración propia.

\subsection{Autopercepción docente de conductas creativas}

Haciendo referencia a las puntuaciones obtenidas por los docentes y especialistas, en la figura 2 se muestra como los primeros tienen una mayor consideración en cuanto al desarrollo creativo del centro, con 3,9 puntos, mientras que los especialistas obtuvieron una puntuación de 3,66. Tanto los docentes como los especialistas consideran que la categoría que menos aporta al desarrollo creativo del centro es la relativa a la Conciencia Creativa de centro (0,75 y 0,53 puntos respectivamente).

Por otra parte, ambos grupos consideran que el principal aporte creativo al centro viene determinado por el Potencial Humano del centro, con unas puntuaciones que van desde 1,95 para los docentes hasta 1,81 para los especialistas.

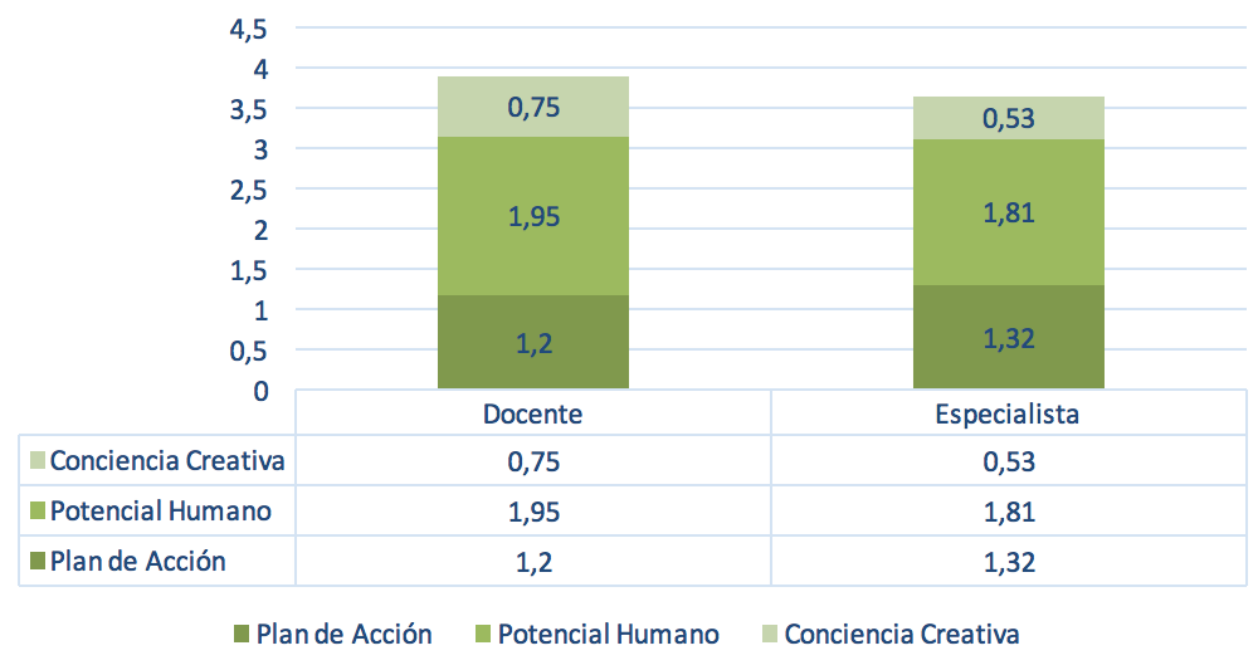

Figura 2. Resultados cuestionario Autopercepción docente Fuente: Elaboración propia.

\subsection{Percepción del Alumnado sobre el profesorado.}


Se han encontrado diferencias significativas $(\mathrm{F}=8,659, p=0,016)$ entre el $1^{\circ}$ Ciclo $(3,01)$ y el $3^{\circ}(2,75)$ con respecto a la puntuación obtenida por el centro. El $2^{\circ}$ Ciclo tiene una puntuación intermedia entre las anteriores, con 2,83. Cabe destacar que a medida que avanzamos de ciclo disminuye la percepción del desarrollo creativo del centro.

Como se representa en la figura 3 la categoría relativa al Potencial Humano del centro es la que más puntuación obtiene en los tres ciclos (1,72, 1,65 y 1,52 puntos respectivamente). $\mathrm{Al}$ igual que en el apartado anterior, a medida que aumentamos de ciclo esta puntuación se ve rebajada.

Los dos primeros Ciclos coinciden en que la variable que menos aporta al desarrollo creativo del centro es la relativa a la Conciencia Creativa del centro. Mientras que en el $3^{\circ}$ Ciclo la categoría que menos puntuación obtuvo fue la referida a los Planes de Acción del centro.

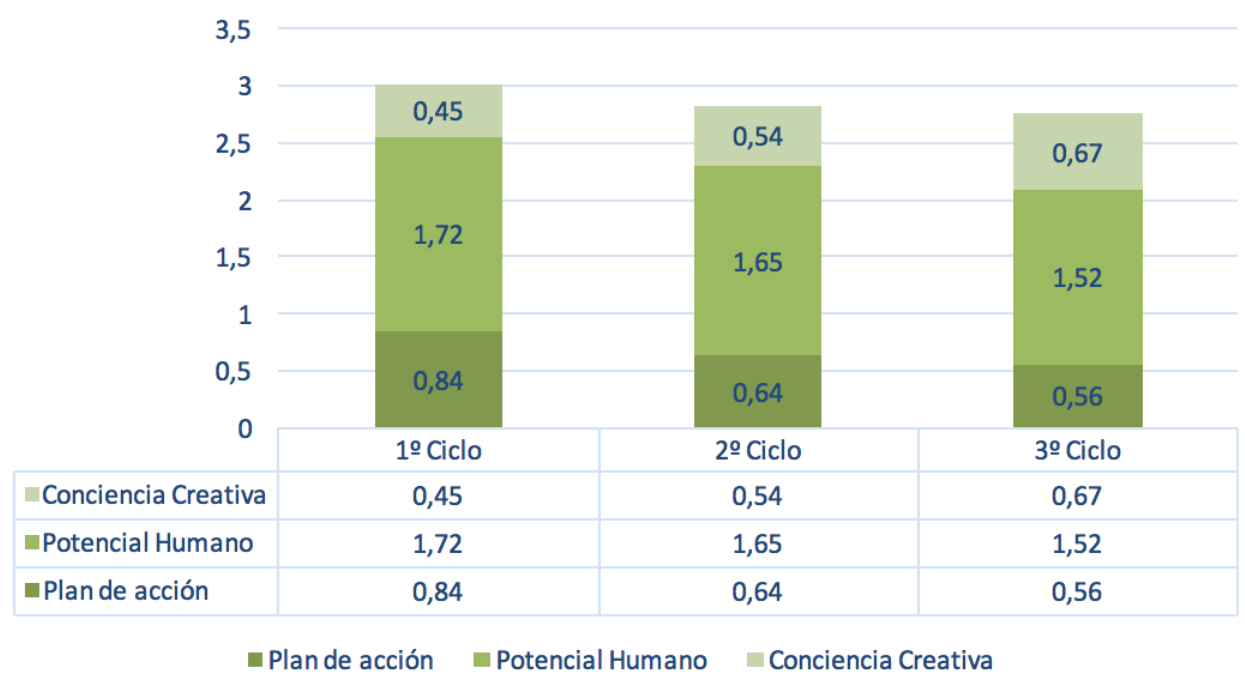

Figura 3. Resultados sobre el cuestionario Percepción del alumnado sobre el profesorado Fuente: Elaboración propia.

La percepción que tiene el alumnado sobre la creatividad docente es menor que la autopercepción docente. Hasta cierto punto tiene sentido por cuanto el docente se autovalora en creatividad al considerarla como valor educativo y social. El alumnado, en cambio, percibe sus conductas más rutinarias y estandarizadas. Forma parte de la autoimagen atribuirnos rasgos que socialmente son considerados. Ello evidencia el papel estimulador del reconocimiento como principio educativo y creativo, como entiende S. Torre (2017) al escribir: "reconocer es recrear".

\subsection{Análisis de los resultados en cuanto al "sexo" de los participantes.}

Tal y como se esperaba, no se han encontrado diferencias significativas en la relación ente el nivel de desarrollo creativo del centro y el sexo de los participantes. En el cuadro 2 se muestran los resultados más relevantes en relación al sexo de los participantes y sus valoraciones. Los hombres del primer grupo obtuvieron una media de 3,75; similar a la obtenida por los varones del equipo directivo $(3,47)$, no siendo esta diferencia significativa $(\mathrm{F}=4,758, p=0.127)$. 
Por su parte, las mujeres del primer grupo obtuvieron una media de 3,82, mientras que las del segundo y tercer grupo 2,92 y 3,33 respectivamente, cuya diferencia tampoco es significativa $(\mathrm{F}=0,143, p=0,707)$.

Cuadro 2. Resultados análisis respecto al sexo de los participantes

\begin{tabular}{lcccccc}
\hline & \multicolumn{2}{c}{ DocENTES } & \multicolumn{2}{c}{ ALUMNADO } & \multicolumn{2}{c}{ EQUIPO DIRECTIVO } \\
\cline { 2 - 7 } & Hombre & Mujer & Hombre & Mujer & Hombre & Mujer \\
\hline Media & 3,75 & 3,82 & 2,78 & 2,92 & 3,47 & 3,33 \\
Desv. típica & 1,40 & 1,19 & 1,50 & 1,37 & 0,94 & 0,57 \\
Máximo & 3,88 & 4,00 & 3,76 & 3,98 & 3,73 & 3,61 \\
Mínimo & 2,89 & 3,21 & 2,55 & 3,03 & 2,95 & 3,24 \\
\hline
\end{tabular}

Fuente: Elaboración propia.

\section{Consideraciones finales}

En líneas generales, el Centro educativo Santo Ángel de La Guarda (Albacete) pone de manifiesto una autopercepción de la creatividad por parte de la Dirección, del profesorado y alumnado por encima del promedio. En el caso del profesorado la media es de 3,78 puntos $-3,75$ (H) y 3,82 (M)-, en el del equipo directivo el valor es de 3,38 puntos -3,47 (H) y 3,33 (M)-. Algo más baja es la media obtenida por el alumnado, 2,86 -2,78 (H) y 2,92 (M)-, lo cual nos permite triangular y contrastar la información obtenida. Esta puntuación puede deberse a una menor comprensión de los conceptos y a ser más exigentes que el propio profesorado en cuanto al desarrollo creativo percibido por el alumnado. Como se muestra en algunos estudios sobre escuelas y aulas creativas, el alumnado suele ser más exigente y crítico que el profesorado (Pujol, Torre, Lorenzo, 2015).

Cabe resaltar que la puntuación más baja vino dada por los miembros del equipo directivo $(1,04)$, quienes dieron mayor importancia a la cultura creativa del Centro y a las metodologías utilizadas (1,22 y 1,12 puntos respectivamente). De esta manera, el principal desafío y oportunidad que afronta el cambio hacia una enseñanza de calidad y creativa está en el profesorado. Un profesorado que tome conciencia de su importancia. Y esto pasa por cambiar el modelo instructivo habitual por un modelo vivencial, crítico y creativo. Según se desprende de la investigación realizada por Torre Frade (2017, p. 49) "al entrar el profesorado en contacto con las prácticas pedagógicas y sus trayectorias formativas, se conectan con sus dimensiones vivenciales, pero también con las invisibles y desconocidas de la formación”. La clave del cambio está en una formación vivenciada.

Falta de conciencia creativa: de acuerdo a las puntuaciones obtenidas, el ámbito que menos aporta al desarrollo creativo es la conciencia creativa, referida a aspectos como la visión, misión, cultura y valores. La realidad actual que enmarca y rodea a nuestras instituciones educativas, presenta escenarios de crisis económica, social, cultural, política, que se constituyen en permanentes desafíos que debemos afrontar si no queremos perder el rumbo. La posición de Torre Frade (2017, p. 48) es clara al respecto al afirmar: "la calidad de la enseñanza no viene dada exclusivamente por los recursos materiales, ni por lo elevado del salario, ni por la ratio alumnos/profesor, sino por la toma de conciencia de la importancia de su papel en la educación".

Los docentes, por su parte, consideraron que las nociones relativas al currículo, a los métodos de trabajo implantados y la evaluación son las que más necesitan ser reformadas para un desarrollo creativo de la institución. No valoran los intereses del alumnado, entendiendo que ha de prevalecer la finalidad de proporcionarles una determinada cultura. 
En tal sentido estamos ante un modo de pensar que dista mucho de una actitud creativa (Menchén, 2015). Perciben la creatividad como valor, pero no como elemento integrante del currículo, aspecto que queda destacado al triangular con información con la percepción del alumnado. Respecto a la manera de enseñar, no se suelen contemplar específicamente unos principios metodológicos, sino que se parte de la convicción de que basta con un buen dominio de los conocimientos disciplinares, aspecto que coincide con los resultados de la investigación de Torre Frade (2017).

No se encontraron diferencias significativas en la autopercepción del Centro en función del sexo. Ambos grupos, varones y mujeres, están de acuerdo en que el desarrollo creativo del centro es bueno, pero que puede ser mejorado en aspectos como el desarrollo de la creatividad a nivel organizativo y de metodología de enseñanza.

Visto el nivel de partida, sería importante establecer un punto de llegada. Una vez interpretados los resultados a la luz de la teoría y otras investigaciones, sería nuestro deseo que este trabajo fuera un punto de partida para seguir profundizando en la creatividad escolar. No son pocos los autores que escriben sobre la creatividad en el aula, pero precisamos polinizar e internalizarla en el Proyecto educativo de Centro, en la organización de espacios, tiempos y contenidos, en las estrategias didácticas y en el sistema de evaluación. Como ya mencionamos anteriormente, los Proyectos creativos ecoformadores (PCE), los diálogos analógicos creativos (DAC) descritos por Torre (2014), el trabajar con Proyectos, el aprendizaje cooperativo, la implicación de la comunidad educativa, la educación desde y para la vida, ... son opciones que ya vienen utilizándose en muchas escuelas de España e Iberoamérica. Hay que abrir el aula, cambiar la metodología poniendo el foco en el aprendizaje y desarrollar una nueva Conciencia basada en valores humanos, sociales, medioambientales y transcendentes como afirman Torre y Moraes (2006), quienes proponen re-encantar la educación mediante procesos de sentipensar; esto es, poner a trabajar conjuntamente pensamiento, emoción y acción.

La escuela creativa debe aceptar el reto de romper con el modelo educativo reproductivo, estandarizado, y basado en el rendimiento académico, y apostar por el desarrollo humano que implica la creatividad; diversificar y actualizar las alternativas metodológicas; desarrollar proyectos innovadores motivantes. Todo esto demanda que el docente se forme y profesionalice como un mediador creativo con fundamentos, con estrategias y con experiencias y vivencias en la práctica. Las escuelas innovadoras y creativas serían los espacios ideales para la praxis docente, tanto inicial como permanente. Para ello es preciso formar maestros con conciencia creativa. Si el docente es capaz de potenciar la originalidad y el talento en sus alumnos, afirma Ken Robinson (2015), entonces habrá logrado algo tremendamente importante: superar el modelo industrial basado en la homogeneización del producto.

\section{Referencias}

Aiken, L. (1996). Test psicológicos y de evaluación. Ciudad de México: Prentice Hall Hispanoamericana.

Costa, G., Lorenzo, N. y Dantas, T. (2016). Inovação e educação. Formação docente e experiências criativas. Ijuí: Unijuí.

De Miguel, M. (2006). Modalidades de enseñanza centradas en el desarrollo de competencias. Oviedo: Servicio de Publicaciones de la Universidad de Oviedo. 
Dispenza, J. (2014). El placebo eres tú. Descubre el poder de tu mente. Barcelona: Urano.

Eisenhardt, K. M. (1989). Building theories from case study research. Academy of Management Review, 14(4), 532-550. https://doi.org/10.5465/amr.1989.4308385

Furlanetto, E. (2011). Formação docente transdisciplinar. O encontro coma experiência. En S. Torre, M. Zwirewicz y E. Furlanetto (Coords.), Formação docente e pesquisa transdisciplinar (pp. 91-132). Santa Catarina: Nova Letra.

Gómez, W. y Machado, A. (2010). Organizaciones educativas creativas bajo una perspectiva humanizante. Revista Ciencias de la Educación, 20(35), 95-121.

Lipton, B. (2014). El efecto de luna de miel. Madrid: Palmyra.

Menchen, F. (1998). Descubrir la creatividad. Madrid: Ediciones Pirámide.

Menchen, F. (2015). La necesidad de escuelas creativas. Madrid: Díaz de Santos.

Moraes, M. C. (2004). Pensamento eco-sistémico. Petropolis: Vozes.

Pitz, J., Silva, V. y Silva, A. (2017). Projetos criativos ecoformadores na educação básica. Santa Catarina: Nova Letra.

Pujol, M. A., Torre, S. y Lorenzo, N. (2015). Escuelas y aulas creativas. Reflexiones y experiencias. Barcelona: Universitat de Barcelona.

Robinson, K. y Aronica, L. (2015). Creative schools. Nueva York, NY: Viking \& Penguin.

Torre, G. y Rodríguez, P. (2009). Autorrealización, creatividad y formación de profesores. Educación y Futuro. Revista de Investigación Aplicada y Experiencias Educativas, 5(2). 71-89.

Torre, S. (2012). Instituciones educativas creativas. Instrumento para valorar el desarrollo creativo de las instituciones creativas (VADECRIE). Almería: Círculo Rojo.

Torre, S. (2014). Aprender dialogando. Diálogos analógicos creativos. Almería: Círculo Rojo.

Torre, S. (2017). Polinizando mi vida. La trayectoria vital de un profesor. Almería: Círculo Rojo.

Torre, S. y Violant, V. (2016). O movimento das escolas criativas: Enraizando no terreno de uma educação transformadora. En G. Costa, N. Lorenzo y T. Dantas (Coords.), Inovação e Educação. Formação docente e experiências criativas (pp. 15-34). Ijuí: Unijuí.

Torre, S. y Moraes, M. C. (2006). Sentipensar. Málaga: Aljibe.

Torre, S. y Moraes, M. C. (2011). Decálogo sobre tansdisciplinariedad y ecoformación. En S. Torre, M. A. Pujol y M. C. Moraes (Coords.), Documentos para el cambio (pp. 47-63). Almería: Círculo Rojo.

Torre, S. y Zwierewicz, M. (2009). Uma escola para o século XXI. Escolas criativas e resiliência na educação. Florianópolis: Editora Insular.

Torre Frade, A. M. (2017). El pensamiento del profesorado en ciclos formativos de turismo respecto a la labor docente. International Journal of World of Turism, 4(7), 37-54.

Welch, S. y Comer, J. (1988). Quantitative methods for public administration. Techniques and applications. Chicago, IL: Dorsey Press. 


\title{
Anexo 1. Cuestionario de Autopercepción Docente
}

\author{
Saturnino de la Torre y João Henrique Suanno
}

- Institución:

- Ciudad:

- Cargo:

- Curso:

- Ámbito o Especialidad:

- Años de docencia:

- E-mail (para informar):

- Sexo $\mathrm{M} / \mathrm{F}$ :

El presente cuestionario pretende facilitar la toma de conciencia y auto percepción como docente. Se trata de marcar el nivel de frecuencia de aquellas actuaciones y creencias conforme a la siguiente escala

Es habitual (A); Frecuentemente $(\boldsymbol{B})$; Ocasionalmente $(\boldsymbol{C})$; Nunca o casi nunca $(\boldsymbol{D})$

Procura no dejarte llevar por lo deseable o bien visto. Sé realista y sincero, pues en ello está la mejora.

1. Creo en mi papel como formador de personas

2. Procuro saber lo que los alumnos piensan, sienten y sus valores

3. Consigo entusiasmar a los estudiantes en proyectos que van más allá del aula y la asignatura

4. Amplio la conciencia del alumnado en sus relaciones con los otros, la naturaleza, el planeta

5. Creo que la educación tiene una función transformadora de personas y de la sociedad

6. Consigo que, en general, el alumnado tenga la sensación de que el tiempo pasa rápido en mis clases

7. Conecto la enseñanza, mis explicaciones, con la vida, con los problemas y la realidad actual

8. Busco y valoro lo nuevo, diferente e ingenioso. Actúo con actitud innovadora en el aula

9. Hago pensar, sentir y actuar. Los contenidos están al servicio de la formación de la persona.

10. Ayudo a los estudiantes a desarrollar la conciencia de valores ambientales en su vida personal.

11. Doy más importancia al desarrollo de las capacidades y actitudes de los alumnos que a los contenidos

12. Procuro saber cuáles son las preocupaciones, gustos y aspiraciones de mis alumnos

13. Tengo facilidad para dialogar, entusiasmar e implicar a los alumnos en las actividades que propongo

14. Considero los problemas como una fuente de aprendizaje

15. Estoy abierto al cambio conectando lo conocido con lo desconocido o que está por conocer

16. Mantengo conversaciones con los alumnos sobre cuestiones ajenas a la asignatura

17. Suelo poner en clase ejemplos de la vida y por eso perciben lo que aprenden como algo útil

18. Me atrae lo nuevo, diferente o ingenioso

19. Doy tanta importancia a los conocimientos como a las emociones y relaciones humanas

20. Procuro desarrollar valores como la autonomía, el esfuerzo, la colaboración, la amistad, el amor.

21. Tengo la convicción de que la educción es la palanca para transformar la sociedad 
22. Reconozco los potenciales que tiene cada uno de los estudiantes, creo en ellos y los estimulo

23. Vivo la enseñanza con entusiasmo y lo trasmito a los alumnos

24. Doy sentido a cuanto ocurre o sucede en mi entorno y lo conecto con la asignatura

25. Hablo con los alumnos sobre hechos cotidianos y sacamos conclusiones para su formación

26. Sorprendes al estudiante con preguntas estimulantes despertando su interés y curiosidad

27. Trasmites vitalidad y optimismo, y si algo no sale como uno desea, buscas el lado positivo

28. Te consideras una persona original y creativa y lo proyectas en tu actividad docente

29. Promueves aprendizajes integrados utilizando estímulos sensoriales, emocionales, diferentes lenguajes

30. Procuro desarrollar en los estudiantes valores como respeto, tolerancia, igualdad, justicia

31. Tengo en consideración las situaciones emergentes, imprevistas y momentos de especial relevancia

32. En la evaluación de los aprendizajes, utilizo recursos e instrumentos diversificados con el fin de contrastar y triangular la información recibida.

33. Promuevo el aprendizaje autónomo, por descubrimiento: inventan, construyen, indagan

34. Planteo la evaluación como una oportunidad para el análisis, la revisión y el reconocimiento de potenciales, talentos y logros.

35. Propongo tareas en las que los alumnos utilizan funciones de ambos hemisferios y del integrado

36. Intento sacar lo mejor de cada persona, sus potenciales y atributos para los que tiene una mejor predisposición.

37. Pongo de manifiesto que mi papel como educador-a va más allá de enseñar contenidos curriculares

38. Entiendo que la evaluación no es un tema de control sino de valoración

39. Procuro transformar los problemas en oportunidades buscando la mejora siempre que puedo

40. Dejo huella. Percibes que tus ex-alumnos te recuerdan años después con satisfacción 


\section{Anexo 2. Cuestionario de Percepción del Alumnado sobre Profesorado}

Saturnino de la Torre

- Colegio:

- Ciudad:

- Curso:

- Sexo M/F:

- Fecha.

- Profesorado Tutor/profesorado del curso:

- Puedes identificarte con la fecha de tu nacimiento:

El presente cuestionario busca conocer la percepción del alumnado sobre la actuación del profesorado de su curso en su función docente y estimuladora de la creatividad. Se trata de marcar el nivel de frecuencia con que ocurren en el aula determinadas actuaciones y creencias docentes.

\section{Es habitual $(\boldsymbol{A})$; Frecuentemente $(\boldsymbol{B})$; Ocasionalmente $(\boldsymbol{C}) ;$ Nunca o casi nunca $(\boldsymbol{D})$}

Procura no dejarte llevar por lo deseable o bien visto. Sé realista y sincero, pues en ello está la mejora.

\begin{tabular}{|c|c|}
\hline \multirow{2}{*}{\multicolumn{2}{|c|}{$\begin{array}{l}\text { 1. El profesorado que tengo cree que es más importante formar personas que impartir } \\
\text { conocimientos. }\end{array}$}} \\
\hline & \\
\hline \multicolumn{2}{|l|}{ 2. Se preocupa por saber lo que los alumnos pensamos, sentimos y nos gusta. } \\
\hline \multirow{2}{*}{\multicolumn{2}{|c|}{$\begin{array}{l}\text { 3. Consigue entusiasmarnos en proyectos que van más allá del aula y la asignatura. } \\
\text { 4. Nos hace ver la importancia de las relaciones con los otros, la conservación de la naturaleza, } \\
\text { el planeta. }\end{array}$}} \\
\hline & \\
\hline \multicolumn{2}{|l|}{$\begin{array}{l}\text { 5. Cree que la educación es transformadora de personas y de la sociedad y no sólo } \\
\text { instrucción. }\end{array}$} \\
\hline \multicolumn{2}{|l|}{ 6. Consigue que tengamos la sensación de que el tiempo pasa rápido en sus clases. } \\
\hline \multicolumn{2}{|l|}{$\begin{array}{l}\text { 7. Pone ejemplos en sus explicaciones de la vida, comenta noticias y lo que pasa en la } \\
\text { realidad. }\end{array}$} \\
\hline \multicolumn{2}{|l|}{ 8. Intenta ser innovador en sus clases y sorprendernos con cosas nuevas. } \\
\hline \multicolumn{2}{|l|}{$\begin{array}{l}\text { 9. Nos hace pensar, sentir y actuar. (La persona es más importante que los resultados de los } \\
\text { exámenes). }\end{array}$} \\
\hline \multicolumn{2}{|l|}{ 10. Nos ayuda a ver la importancia de conservar la naturaleza y cuidar el medio ambiente. } \\
\hline \multicolumn{2}{|l|}{$\begin{array}{l}\text { 11. Cuida tanto del desarrollo de las capacidades y actitudes como de los contenidos de la } \\
\text { asignatura }\end{array}$} \\
\hline \multicolumn{2}{|l|}{ 12. Intenta conocer las preocupaciones, gustos y aspiraciones de los alumnos. } \\
\hline \multicolumn{2}{|l|}{ 13. Facilita el diálogo en clase implicando a los alumnos las tareas de aprendizaje. } \\
\hline \multicolumn{2}{|l|}{ 14. Considera los problemas como una fuente de aprendizaje. } \\
\hline \multicolumn{2}{|l|}{$\begin{array}{l}\text { 15. Está abierto a cosas nuevas, relacionando lo conocido con lo desconocido para el } \\
\text { alumnado. }\end{array}$} \\
\hline \multicolumn{2}{|l|}{ 16. Mantiene conversaciones con los alumnos sobre cuestiones ajenas a la asignatura. } \\
\hline \multicolumn{2}{|l|}{$\begin{array}{l}\text { 17. Suele poner en clase ejemplos de la vida y por eso perciben lo que aprenden como algo } \\
\text { útil. }\end{array}$} \\
\hline \multicolumn{2}{|l|}{ 18. Recurre como estrategia de enseñanza a a algo sorprendente, diferente o ingenioso. } \\
\hline \multicolumn{2}{|l|}{$\begin{array}{l}\text { 19. Da importancia a la parte emocional y humana de los alumnos, sus problemas y } \\
\text { conflictos. }\end{array}$} \\
\hline 20. Promueve valores personales como la autonomía, el esfuerzo, la colaboración, la & \\
\hline
\end{tabular}


21. Nos dice que con la Educación podemos cambiar la sociedad en la dirección que deseamos.

22. Reconoce los talentos de sus alumnos, cree en ellos y los anima a desarrollarlos.

23. Vive la enseñanza con entusiasmo y los alumnos lo notamos.

24. Da sentido a cuanto ocurre o sucede en la actualidad y lo conecta con la asignatura.

25. Habla con los alumnos sobre hechos cotidianos y saca conclusiones para la educación.

26. Sorprende a sus alumnos con preguntas estimulantes, despertando su interés y curiosidad.

27. Trasmite vitalidad y optimismo, y si algo no sale como desea, busca el lado positivo.

28. Se le ve una persona original y creativa y lo muestra en su modo de enseñar y relacionarse.

29. Promueve aprendizajes significativos, que dejan huella, utilizando estímulos sensoriales y emocionales.

30. Trasmite a sus alumnos valores sociales como respeto, tolerancia, igualdad, justicia...

31. Para él/ella, lo más importante es ayudar y acompañar al alumno a desarrollar sus talentos.

32. Utiliza el reconocimiento para motivar a alumnado a superarse y aprender por sí mismo.

33. Está convencido de que la pasión y entusiasmo por educar es clave en la enseñanza.

34. No le importa decir que su papel es Educar más que enseñar contenidos curriculares.

35. Procura transformar los problemas en oportunidades buscando siempre lo mejor para sus alumnos

36. Deja huella. Sabe que tus ex -alumnos te recuerdan años después con cariño.

37. Las dificultades no le desaniman, sino por el contrario saca algún provecho de ellas.

38. Practica en clase y fuera el aprendizaje autónomo y por descubrimiento: inventar, construir, indagar.

39. Propone tareas que obligan a pensar y respuestas que no están en el libro, insólitas y poco habituales.

40. Da importancia a las creencias, actitudes y valores humanos y sociales de los alumnos. 


\title{
Anexo 3. Cuestionario de Autopercepción Equipo Directivo
}

\author{
José Luis Marcilla Cebrián y Saturnino de la Torre
}

- Institución:

- Ciudad:

- Cargo:

- Curso:

- Ámbito o Especialidad:

- Años de docencia:

- E-mail (para informar):

- $\operatorname{Sexo} \mathrm{M} / \mathrm{F}$ :

El presente cuestionario pretende facilitar la toma de conciencia y auto percepción como miembro del equipo directivo. Se trata de marcar el nivel de frecuencia de aquellas actuaciones y creencias conforme a la siguiente escala:

Es habitual (A); Frecuentemente $(\boldsymbol{B}) ;$ Ocasionalmente $(\boldsymbol{C}) ;$ Nunca o casi nunca $(\boldsymbol{D})$

1. Demostramos un perfil creativo en el contexto de nuestra labor directiva

ESCALA

2. Proponemos unas metas explícitas y compartidas que generan compromiso entre los miembros.

3. Promovemos metas que llevan implícitas gratificación y reconocimiento.

4. El equipo directivo sabe compartir y consultar las acciones que se llevan a cabo como organización.

5. Procuramos que dentro de la planificación del centro escolar se realicen acciones que potencien la creatividad de profesores y alumnos.

6. Demostramos saber adaptarnos a los cambios y necesidades del entorno.

7. Buscamos, en todo momento, formas de autorregular la toma de decisiones, en su ejecución y evaluación.

8. Promovemos la armonía estructural, interpersonal y de funcionamiento entre los miembros del equipo directivo.

9. Aseguramos la difusión de información acerca de las acciones orientadas a desarrollar la creatividad en docentes y alumnos.

10. Consideramos que la educación tiene una función transformadora de personas y de la sociedad

11. Logramos mantener un clima de relaciones fluidas, de comunicación fácil y seguridad psicológica.

12. Poseemos capacidades para reconocer las debilidades y potenciar las fortalezas.

13. Establecemos relaciones y vínculos con otras instituciones con las que compartimos encuentros, redes o proyectos.

14. Tomamos decisiones fundamentadas, y consideramos enfoques alternativos, a fin de desarrollar la creatividad en docentes y alumnos.

15. Consideramos las adversidades como fuentes de conocimiento y oportunidades de crecimiento

16. Promovemos, entre los docentes, altas expectativas de desarrollo creativo en los estudiantes.

17. Aprovechamos todas las potencialidades de los profesionales que intervienen en el proceso educativo. 
18. Obtenemos recursos adicionales (humanos, financieros, materiales y técnicos) para apoyar las prácticas docentes que buscan potenciar la creatividad de los estudiantes

19. Buscamos lo mejor para nuestra escuela facilitando recursos, realizando cursos o seminarios, etc.

20. Promovemos el compromiso y la participación del personal docente en cada una de las actividades que busquen mejorar la creatividad de los alumnos

21. Tomamos en especial consideración la formación de nuestro equipo docente. Tienen planes de formación continuada.

22. Proporcionamos instancias de perfeccionamiento docente para el aprendizaje de estrategias que favorezcan la creatividad de los estudiantes.

23. Valoramos la diversidad de culturas, creencias y diferencias personales como riqueza y no como discriminación.

24. Garantizamos el desarrollo de instancias de análisis y reflexión sobre la relación entre las prácticas pedagógicas creativas y los resultados

25. Promovemos, entre los docentes, la implementación de estrategias de enseñanza creativas. Estamos abiertos a las nuevas miradas pedagógicas.

26. Promovemos, entre los estudiantes, altas expectativas en cuanto al desarrollo de sus capacidades creativas.

27. Generamos tiempos para determinar criterios y diseñar instrumentos de evaluación que permitan retroalimentar las prácticas creativas de los docentes

28. Aseguramos la disponibilidad de recursos pedagógicos que favorezcan el desarrollo de la creatividad de profesores y alumnos.

29. Nos ocupamos de las necesidades del personal docente, apoyando y motivando el desarrollo de su perfil creativo.

30. Establecemos un sistema de difusión de las actividades y proyectos del establecimiento orientados a desarrollar la creatividad de los estudiantes.

31. Diseñamos actividades orientadas a dar a conocer a la comunidad escolar el potencial creativo de sus estudiantes.

32. Establecemos un sistema de difusión de las actividades y proyectos del establecimiento orientados a desarrollar la creatividad de los estudiantes.

33. Estamos abiertos a recibir comentarios, ideas y sugerencias sobre cómo potenciar la creatividad en docentes y alumnos.

34. Promovemos la incorporación de las nuevas tecnologías a las aulas.

35. Diseñamos actividades orientadas a dar a conocer a la comunidad escolar el potencial creativo de sus estudiantes.

36. Generamos instancias de diálogo, con padres y apoderados, que permitan otorgarles estrategias para el desarrollo de la creatividad de sus hijos.

37. Estimulamos la participación de los padres y/o apoderados en el desarrollo de la creatividad de sus hijos.

38. Consideramos que estamos al frente de una institución saludable.

39. Evaluamos la relación escuela-familia, en cuanto a la participación de los padres en el desarrollo de la creatividad de sus hijos.

40. Promovemos el desarrollo de un clima interno propicio a la cultura innovadora donde las ideas y las funciones puedan prosperar en un clima de mejora continuo.

\section{Breve CV de los autores}

\section{Saturnino de la Torre}

Catedrático emérito de la Universidad de Barcelona. Ha sido Coordinador del Grupo de Investigación y Asesoramiento Didáctico (GIAD). Impulsor del Seminario de Cine Formativo, miembro fundador de la Fundación para el desarrollo de la Creatividad de UNESCO (1990), Promotor de la Red Internacional de Creatividad (1996), de ASOCREA (2000) y Presidente de la misma en el período inicial (2001-2006). Coordinador de la Red Internacional de escuelas creativas (RIEC). Ha investigado sobre innovación educativa; sobre creatividad, desarrollo y evaluación; sobre estrategias didácticas innovadoras y 
creativas y actualmente sobre Escuelas creativas y la Adversidad creadora desde una mirada compleja y transdisciplinar. Encabeza más de 50 libros, 160 capítulos, 60 artículos, 22 prólogos, 33 entrevistas, 75 tribunales de tesis. Página web: www.saturninotorre.es. ORCID ID: https://orcid.org/o000-0003-3898-0465. Email: sentipensar@yahoo.es

\section{José Luis Marcilla Cebrián}

Profesor de Inglés y Español en colegio de Educación Primaria Skola Klic S.R.O. en Ceská Lípa (República Checa). Ha realizado Máster en formación de profesores de español como lengua extranjera (ELE) y especialista en creación de materiales educativos. En 2015, finalizó Máster en Formación del Profesorado para alumnos con altas capacidades en la UCLM. Actualmente colabora como preparador de monitores juveniles en proyectos Erasmus + y es mentor de voluntarios en proyectos EVS (European Volunteering Service) en República Checa. Ha participado en la creación, seguimiento y evaluación de intercambios juveniles centrados en la enseñanza por medio métodos alternativos a los tradicionales y facilitador de enseñanza primaria a minorías étnicas y culturales. ORCID ID: https://orcid.org/0000-0003-2737-032X. Email: jlmc_9@hotmail.com

\section{Ana María de la Torre Frade}

Profesora de Ciclos formativos de turismo desde el año 2004 en l'Escola d'Hoteleria i Turisme de Barcelona, habiendo impartido más de 15 asignaturas así como cargos de responsabilidad dentro del centro. Graduada en Turismo en la escuela universitaria CETT, Máster en Innovación Turística en la UB (2011). Diploma de Estudios Avanzados (DEA) y actualmente realizando la tesis doctoral en la Universidad de Girona sobre Innovación turística. Tiene recientes publicaciones sobre el Pensamiento del profesor de Ciclos formativos en la revista International Journal of World of Tourism (2017). Participa en el proyecto "Qualifica't", un proyecto de innovación educativa gestionado por "Ensenyament " y forma parte del Equipo de Innovación y mejora de Fp Dual. ORCID ID: https://orcid.org/0000-0003-1612-9426. Email: atorre47@xtec.cat 